\title{
MULTIPLE SCATTERING AND NEUTRALIZATION ASPECTS OF LOW ENERGY NOBLE GAS IONS INCIDENT ON A Cu (110) CRYSTAL
}

\author{
A.G.J. DE WIT, G.A. VAN DER SCHOOTBRUGGE and J.M. FLUIT \\ Laboratory of Experimental Physics, \\ University of Utrecht, Utrecht, The Netherlands
}

\begin{abstract}
Experiments have been done with $1 \mathrm{keV} \mathrm{Ne}^{+}$ions bombarding a $\mathrm{Cu}(110)$ single crystal in a (111) plane. From the measured energy spectra of the scattered ions the minimum and maximum scattering angles for multiple scattering on the surface are determined. These minimum and maximum scattering angles were also calculated using a computer model. The parameters for an interatomic potential function between ion and metal atom are determined by comparison of the experimental and calculated scattering angles. A neutralization model for an energetic ion at an atomic surface is given. The reliability of this model is tested by comparison of the results of computer calculations and the measured peak intensity distributions as a function of the scattering angle.
\end{abstract}

\section{Introduction}

Analysis of the energy spectrum of noble gas ions scattered from the clean surface of a metal single crystal shows two peaks for a certain range of scattering angles, when the plane of incidence is along a closed packed surface chain. The low energy peak can be identified with a mainly binary collision process, the other one with a multiple collision process. The energies corresponding to these peaks depend on the potential function between the collision partners, the angle of incidence, the scattering angle, the mass ratio and the distance between the surface atoms in the plane of incidence (in the case of multiple scattering) $[1,2]$. When at fixed angle of incidence, primary energy and mass ratio the relative energy of the peaks is plotted as a function of the scattering angle, the so-called multiple scattering loops are obtained (fig. 1b).

Fig. 1a shows measured energy spectra for $\mathrm{Ne}^{+}(1 \mathrm{keV}) \rightarrow \mathrm{Cu}(110)$, with a (111) plane as the plane of incidence, for different scattering angles. The angle of incidence is always $30^{\circ}$ with respect to the surface of the target. The two peaks approach each other with decreasing scattering angle. At small scattering angles the high energy peak disappears, suggesting that in that case multiple collision processes cannot 
(a)

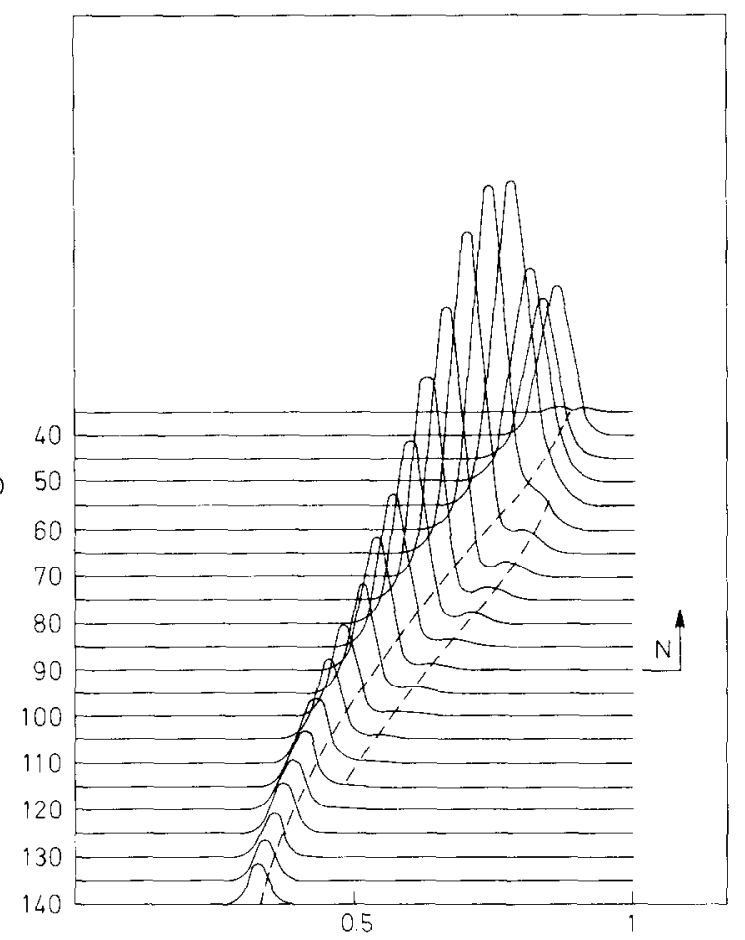

$E / E_{0}$

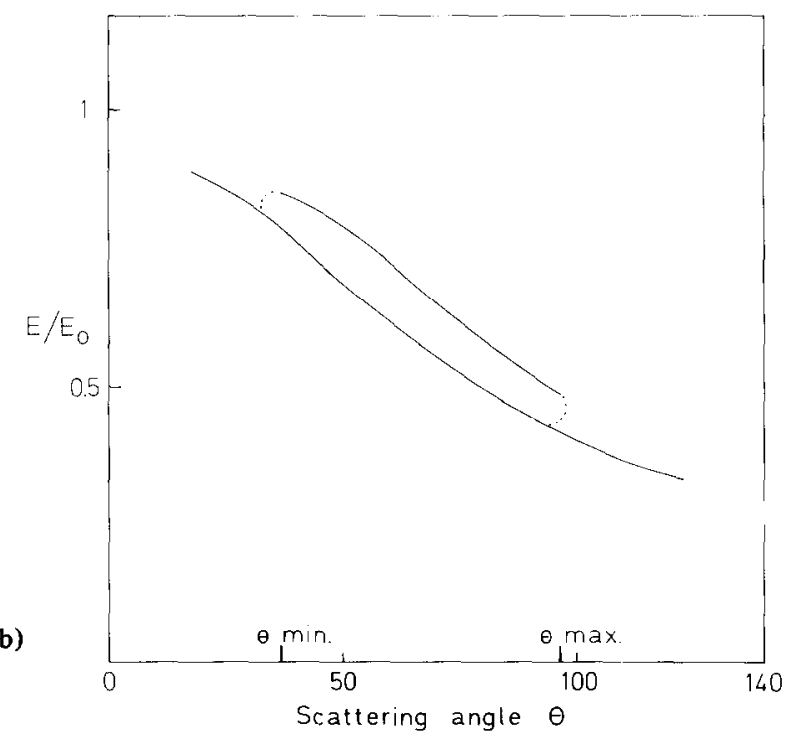

Fig. 1. (a) Energy spectra of $1 \mathrm{keV} \mathrm{Ne}^{+} \rightarrow \mathrm{Cu}(110)$ for different scattering angles $O$ (plane of incidence (111), angle of incidence $\phi=30^{\circ}$ with respect to the surface). (b) The relative energy of the scattering peaks versus scattering angle - taken from (a). 
occur. This is in agreement with reports of Kivilis et al. [3], Yurasova et al. [2], Maskova et al. [4], Heiland et al. [5] and can easily be understood from the fact that at small angles of incidence and for low primary energy of the incoming ions not all possible impact parameters are permitted (surface atomic screening [6]. Analogous arguments explain the existence of a maximum scattering angle above which no scattering can occur. Hig. lb shows the relative energy of the peaks as a function of the scattering angles. Such a multiple scattering loop is limited within $\theta_{\max }$ and $\theta_{\min }$.

Computer calculations have pointed out that $\theta_{\min }$ and $\theta_{\max }$ depend on the assumed potential function for the interaction between a noble gas particle and the atoms in the metal surface $[2,3]$. The best fit of the experimental results and a computer model is obtained by varying the parameters of the potential function between noble gas particles and surface atoms [17]; mostly the Born-Mayer potential function has been used: $V(r)=A \mathrm{e}^{-b r}$. However, the form of the potential function is not the only factor determining the shape of the energy spectra. The following processes have to be included:

(i) Neutralization processes near the metal surface. They depend on the distance between the ion and the surface atoms [7-9] and can have an important influence on the peak height.

(ii) Thermal vibrations of the surface atoms. An energy spectrum measured at fixed scattering angle is an average of different microscopic angles of incidence $[10,11]$. Owing to this effect the microscopic angle of incidence varies, hence energy spectra measured at a fixed scattering angle are a superposition of many multiple scattering loops. Moreover thermal vibrations in combination with neutralization processes can give rise to a peak shift (especially of the high energy peak) and also to a change of peak intensity.

(iii) Surface imperfections. Scattering on surface defects (for instance atoms on top of the surface) $[12,13]$ causes an increase of the low energy peak intensity, because single collisions can take place on surface defects with a very low neutralization probability (scattering on foreign atoms is not considered).

These processes, if important, will be included in the discussion of our experimental and calculated results.

Ion reflection spectroscopy as a tool for surface analysis is confronted with the above mentioned difficulties. To make it a reliable and quantitative technique more has to be known about interatomic potentials and relevant parameters in the neutralization processes.

\section{Experimental set-up}

The experimental set-up is schematically shown in fig. 2. A Nier type ion source with an accelerator system produces a noble gas ion beam with a total current in the 
range of $10^{-8}-10^{-6} \mathrm{~A}$. The beam is focussed at the target in a spot of about $2 \mathrm{~mm}$ diameter at normal incidence. The primary energy can be varied between $100 \mathrm{eV}$ and $10 \mathrm{keV}$, with a relative energy spread of $0.1 \%$. The background pressure in the target chamber is about $5 \times 10^{-9}$ torr; the working pressure is $10^{-7}$ torr.

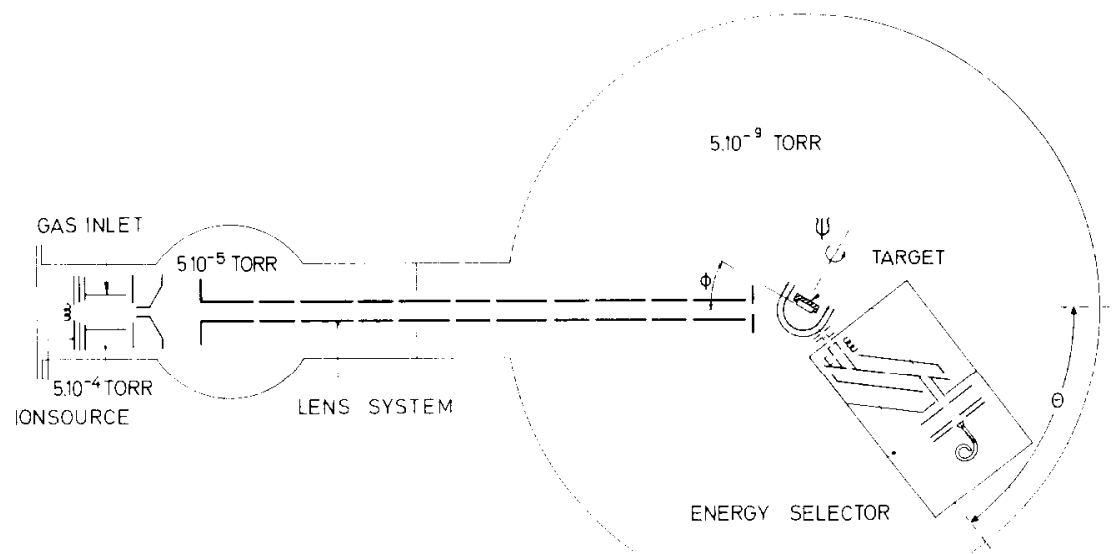

Fig. 2. Sketch of the experimental set-up.

The target can be rotated over $180^{\circ}$ around the normal to the surface. The angle of incidence $\phi$, can be varied between $0^{\circ}$ and $90^{\circ}$. An electrostatic energy analyzer (flat condenser type) can be rotated around the target in the plane of incidence which allows a variation of the angle $\theta$ between the direction of the incident ions and the direction of detection from $0^{\circ}$ to $145^{\circ}$. With this double electrostatic analyzer we can choose and measure the energy spread. Usually we made our experiments with an energy resolution of $\Delta E / E=3.5 \%$. The angular resolution of the analyzer system depends also on the spot size of the ion beam and in our case is given by

$$
\Delta \theta=\operatorname{arctg}\left[0.064 \times \frac{\sin (\theta-\phi)}{\sin \phi}\right]
$$

at FWHM with a maximum of $6.5^{\circ}$ (see also fig. 6). A small electron source in front of this analyzer permits ionization of neutral particles. The mass of the secondary ions can be measured with a time of flight method. A channeltron electron multiplier at the output of the analyzer counts the selected ions.

\section{Experimental results and discussion}

Energy spectra have been measured at different scattering angles and angles of incidence (fig. 1). All the experiments shown here have been done with $1 \mathrm{keV} \mathrm{Ne}^{+}$ions 
bombarding a $\mathrm{Cu}(110)$ single crystal in a (111) plane. The primary ion intensity was $0.2 \mu \mathrm{A} / \mathrm{cm}^{2}$; the total ion dose per energy spectrum was $5 \times 10^{13}$ ions $/ \mathrm{cm}^{2}$. Scattering peaks due to foreign atoms at the surface were not found. Fig. 3 shows plots of the relative energy of the peaks as a function of the scattering angle for different values of the angle of incidence $\phi$ (relative to a direction in the surface).

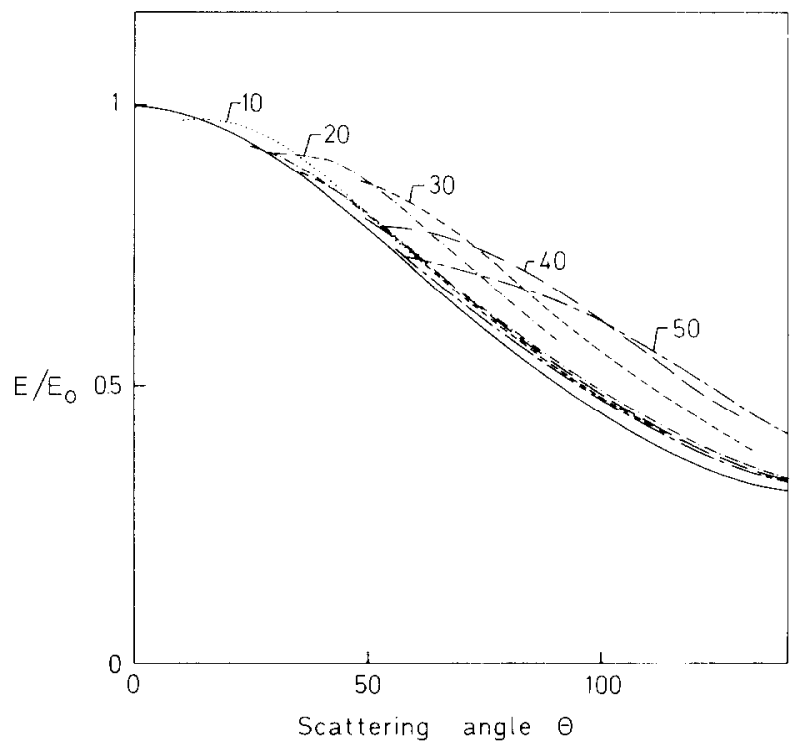

Fig. 3. The relative energy of the scattering peak versus scattering angle $\theta$ for $1 \mathrm{keV} \mathrm{Ne}^{+} \rightarrow \mathrm{Cu}(110)$ at $\phi=10^{\circ}, 20^{\circ}, 30^{\circ}, 40^{\circ}$ and $50^{\circ}$ degrees (full line gives the theoretical relation for single collision of $1 \mathrm{keV} \mathrm{Ne}^{+} \rightarrow \mathrm{Cu}$ ).

It has to be noticed that the left-hand side as well as the right-hand side of the multiple scattering loops are difficult to deduce from measured spectra like those in fig. 1a. However, computer calculations show that especially the outer ends of the loops depend strongly on the potential parameters. So fig. 3 is not very well suited for fitting these parameters. In fig. 4 the peak intensity of the "single" collision peak is plotted as a function of the scattering angle at fixed angles of incidence.

There is, except at $\phi=10^{\circ}$, an obvious kink in the curves at the small scattering angle side. The shape of these curves can be understood if we assume that part of the reflected ions scattered from a smooth surface and another part from surface defects introduced by the bombardment. This assumption is sustained by the influence of ion dose on the peak intensity at small scattering angles. This effect was also found by Heiland et al. [18]. For the ions scattered from defects all scattering angles are possible, while for ions scattered from a smooth surface there is a more or less welldefined range of possible scattering angles. From the distribution of peak intensities (fig. 4) the left hand side of the multiple scattering loops can consequently be determined. 


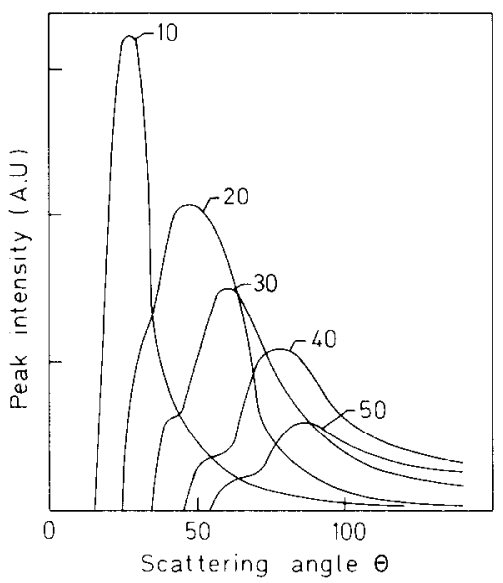

Fig. 4. Peak intensity of the scattering peak of the mainly single scattered ions versus scattering angle $\theta$, for $1 \mathrm{keV} \mathrm{Ne}^{+} \rightarrow \mathrm{Cu}(110)$ at $\phi=10^{\circ}, 20^{\circ}, 30^{\circ}, 40^{\circ}$ and $50^{\circ}$.

In principle it is possible to obtain from fig. 4 the value of the maximum multiple scattering angle $\theta_{\max }$ at low angles of incidence, because at $\theta_{\max }$ the peak intensity must decrease. In our experiments, however, the background of ions scattered from surface defects and the very low peak intensity at large scattering angles made this effect hard to observe.

Experimental results of $1 \mathrm{keV} \mathrm{Ne}^{+} \rightarrow \mathrm{Cu}(110)$ at fixed scattering angle $\theta$, but at variable angle of incidence $\phi$, can give more information about the maximum multiple scattering angle. In fig. 5 the peak intensity of the mainly single collision peak is plotted as a function of the angle of incidence at fixed scattering angle $\left(\theta=60^{\circ}\right.$ and $\theta=80^{\circ}$ ).

Also the peak width is given in this figure. At small angles of incidence very few reflected ions were detected. Increase of the angle $\phi$ causes the yield of reflected ions to rise strongly at $\phi_{1}$. Obviously at angles larger than $\phi_{1}$ scattering of ions by a surface chain becomes possible. Consequently at this angle $\phi_{1}$ the right hand side of the multiple scattering loop ends at a scattering angle $\theta_{1}$ (resp. $\theta_{2}$ ). In the same way one can expect that for angle of incidence $\phi_{2}$ the left hand side of the loop begins at $\theta_{1}$ (resp. $\theta_{2}$ ). From these measurements one can determine the angles of incidence at which the multiple scattering loops begin or end at $\theta_{1}$ (resp. $\theta_{2}$ ). The shape of the curve between $\phi_{1}$ and $\phi_{2}$ can be explained by thermal vibrations [11] and neutralization processes. Reflected ions scattered from surface defects are responsible for the part of the curve outside these angles. 


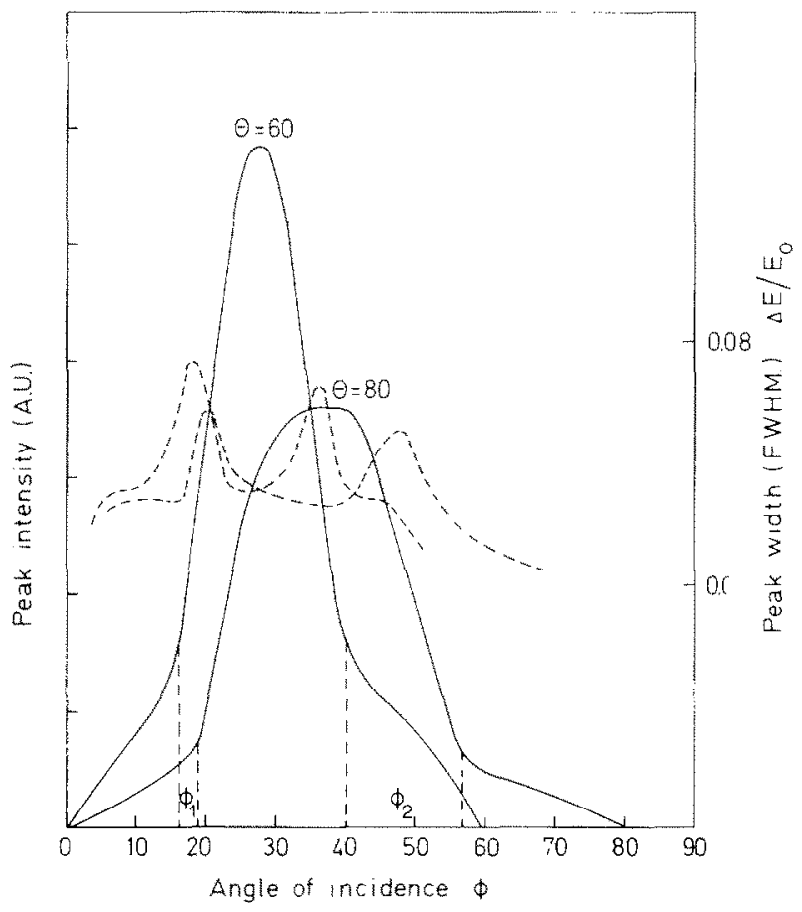

Fig. 5, Peak intensity of the scattering peak of the mainly single scattered ions (full line) versus angle of incidence $\phi$ for $1 \mathrm{keV} \mathrm{Ne} \rightarrow \mathrm{Cu}(110)$ at fixed scattering angles $\left(\theta=60^{\circ}\right.$ and $\left.\theta=80^{\circ}\right)$. Dashed lines give the peak width (FWHM).

\section{Calculations and comparison with the experimental results}

\subsection{Collision process $1 \mathrm{keVNe}^{+} \rightarrow \mathrm{Cu}(110)$ without neutm .ization; interatomic} potential parameters

In the computer model of $1 \mathrm{keV} \mathrm{Ne}^{+} \rightarrow \mathrm{Cu}(110)$ the interatomic potential between the noble gas ion and the metal atoms was approximated by the function $A \exp (-b r)$ ( $A$ and $b$ are constants, $r$ is the distance between ion and atom). From this potential function the force exerted by the metal atoms on the ion was calculated. The equation of motion reads

$$
M_{\text {ion }} \frac{\mathrm{d}^{2} r_{\text {ion }}}{\mathrm{d} t^{2}}=\sum_{i} F_{i},
$$


where $M_{\text {ion }}$ is the mass of the ion, $r_{\text {ion }}$ the position of the ion and $F_{i}$ the force of atom $i$ on the ion. This equation was solved by means of a multistep method of the fourth order with glancing time steps [14]. In these calculations about 20 surface atoms arranged in the first and second surface layer were involved.

In a representative part of the surface a homogeneously distributed set of impact points was chosen. As for the experiments one can plot the relative energy of the scattered particles as a function of the scattering angle and find the multiple scattering loops.

Calculations with this model show that the left-hand (low scattering angle) side of the loops is sensitive for variations in $b$, while the right-hand side is sensitive for $A$ as well as $b$.

The values of $A$ and $b$ were varied around the potential parameters used by Yurasova [2] $\left(A=1.6 \times 10^{-16} \mathrm{~J}, b=5.1 \AA^{-1}\right.$ for $\mathrm{Ne}^{+}$on $\left.\mathrm{Cu}\right)$ and those calculated from the data of Abrahamson [15] $\left\{V(r)_{\mathrm{Nc}->\mathrm{Cu}}=\left[V(r)_{\mathrm{Nc} \rightarrow \mathrm{Nc}} V(r)_{\mathrm{Cu}, \mathrm{Cu}}\right]^{1 / 2}\right.$, $A=1.05 \times 10^{-15} \mathrm{~J}, b=3.67 \AA^{-1}$ for $\left.\mathrm{Ne} \rightarrow \mathrm{Cu}\right\}$. For these values of $A$ and $b$ the minimum angle of multiple scattering, $\theta_{\mathrm{min}}$, was determined at different angles of incidence $\phi$. At small angles of incidence also $\theta_{\text {max }}$ was determined. These values of $\theta_{\min }$ and $\theta_{\max }$ have been plotted in fig. 6 . This figure shows also the experimental values of $\theta_{\min }$ (from fig. 4) and $\theta_{\max }$ (from fig. 5). Comparison with the experimental results shows a better agreement with the parameters taken from Abrahamson than with those used by Yurasova. However, $\theta_{\max }$ from Abrahamson's values is too small. This can be corrected by introducing a larger value of $A$.

\subsection{Collision process $1 \mathrm{keV} \mathrm{Ne} \mathrm{e}^{+} \rightarrow \mathrm{Cu}(110)$ with neutralization; the neutralization parameters}

\subsubsection{Introduction}

The neutralization probability of a reflected ion depends on the trajectory above the surface. Hence the trajectory will affect the peak intensities of the energy distributions of the reflected ions. These peak intensities also depend on the differential scattering cross sections of the surface atoms, which in their turn are dependent on the energy of the incoming ions and the interatomic potential. The Born-Mayer approximation leads to a yield $N \mathrm{~d} \theta$ of particles scattered from one atom in an interval $\mathrm{d} \theta$, decreasing at increasing scattering angle, see fig. 7 . In this figure also the calculated density distribution is plotted as a function of the scattering angle, for $1 \mathrm{keV} \mathrm{Ne}^{+}$reflected from a $\mathrm{Cu}(110)$ surface (angle of incidence $\phi=30^{\circ}$ ). The upper part of the curve originates from particles mainly scattered on one atom, while the lower part originates from particles, which have made mainly two collisions on the surface. These density distributions also depend on the potential parameters. (In fig. 7 Ahrahamson's potential parameters have been used.)

The experimental peak intensity distribution for $\phi=30^{\circ}$, shown in figs. 4 and 8 (curve c) does not agree with the calculated distribution (fig. 7). The experimental curve has to be corrected for particles scattered from surface defects, which give rise 


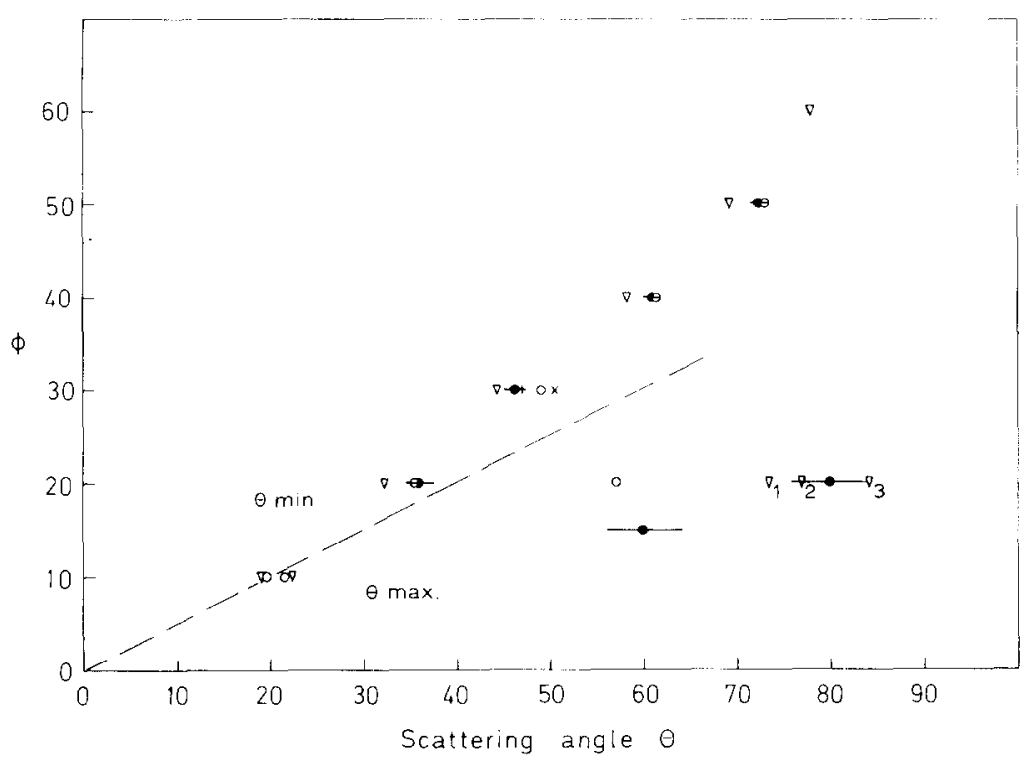

Fig. 6. Relation between angle of incidence $\phi$ and maximum and minimum scattering angles $\left(\theta_{\max }\right.$ and $\left.\theta_{\min }\right)$ for multiple scattered ions $\left[1 \mathrm{keV} \mathrm{Ne}^{+} \rightarrow \mathrm{Cu}(110)\right.$, plane of incidence (111)]. The dots represent experimental results (determined from figs. 4 and 6 ), the remaining symbols are calculated values:

\begin{tabular}{lll}
\hline & $b\left(\AA^{-1}\right)$ & $A(\mathrm{~J}) \times 10^{15}$ \\
\hline$\times$ & 3.2 & 1.05 \\
0 & 3.67 & 1.05 \\
+ & 4.0 & 1.05 \\
$\nabla_{1}$ & 5.1 & 1.8 \\
$\nabla_{2}$ & 5.1 & 1.6 \\
$\nabla_{3}$ & 5.1 & 1.4 \\
$\nabla$ represents & $\nabla_{1}, \nabla_{2}, \nabla_{3}$ together \\
\hline
\end{tabular}

to scattering angles smaller than $\theta_{\min }$. In other words one must subtract from the experimental curve the distribution of particles scattered from one atom. The difference, that still exists, between the corrected experimental curve (fig. 8, curve b) and the calculated curve (of fig. 7) is caused by the fact that neutralization processes were not considered until now in our calculations.

\subsubsection{Computer calculations of neutralization probability}

In addition to the procedure described in section 4.1 , we now solve, simultaneously with the equation of motion, a differential equation, which describes the neutralization 


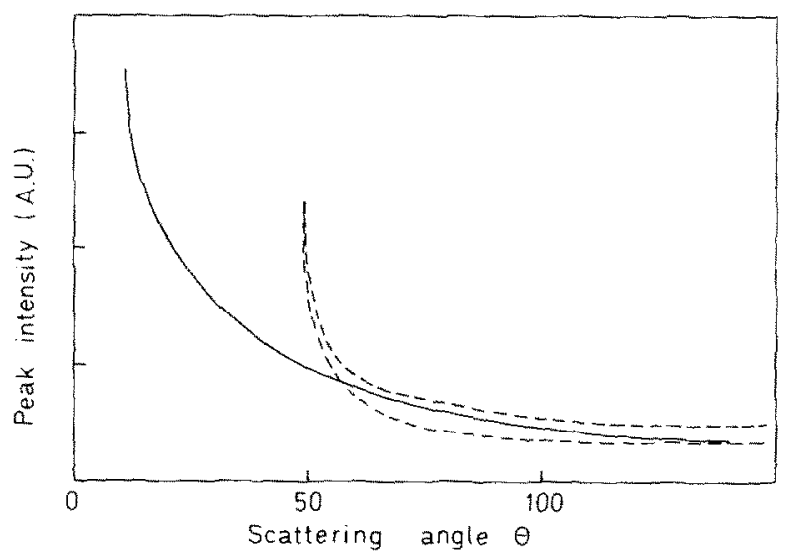

Fig. 7. The density distributions of scattered particles versus scattering angle $\theta$ for $1 \mathrm{keV} \mathrm{Ne} \rightarrow \mathrm{Cu}$. Full line $\mathrm{Ne}^{+} \rightarrow \mathrm{Cu}$, dashed line $\mathrm{Ne}^{+} \rightarrow \mathrm{Cu}(110)$ crystal $\left(\phi=30^{\circ}, A=1.05 \times 10^{-15} \mathrm{~J}, b=3.67 \mathrm{~A}^{-1}\right)$.

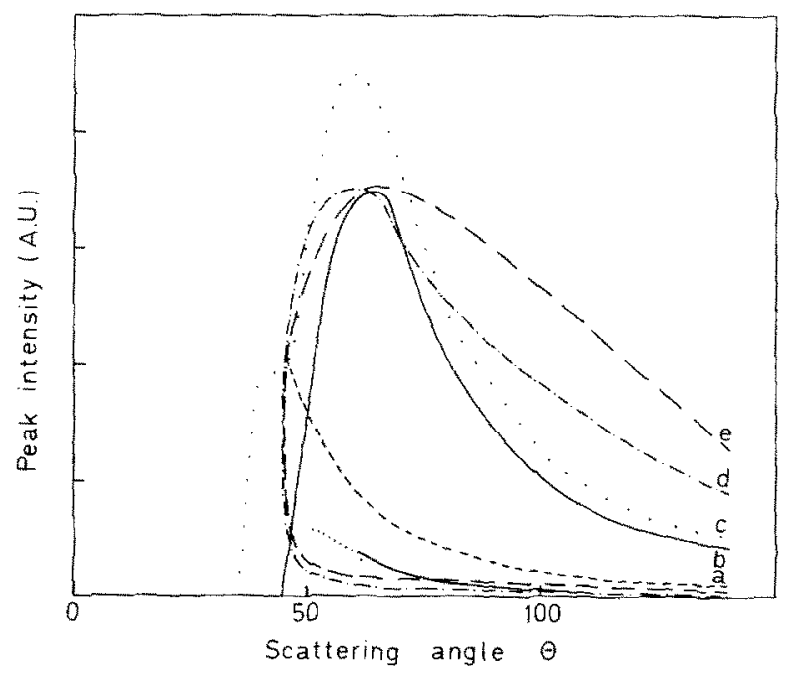

Fig. 8. Peak intensity versus scattering angle $\theta$ for $1 \mathrm{keV} \mathrm{Ne}{ }^{+} \rightarrow \mathrm{Cu}(110)\left(\phi=30^{\circ}\right)$. (a) experimental for ions scattered on surface defects; (b) experimental results corrected for scattering on defects; (c) experimental results (from fig. 4); (d) calculated for $\alpha=2.0 \mathrm{~A}^{-1}$ and $C=9.4 \times 10^{15} \sec ^{-1}$; (e) calculated for $\alpha=1.5 \mathrm{~A}^{-1}$ and $C=3.4 \times 10^{15} \mathrm{sec}^{-1}$.

Curves $d$ and $e$ are normalized with respect to the maximum of $b$. 
probability of the scattered particle. An expression for the neutralization probability of an ion in an interval $\mathrm{d} s$ ( $s=$ distance to the surface) often used $[16,8,9]$ is:

$$
f(s) \mathrm{d} s=C \exp (-\alpha s) \mathrm{d} s,
$$

where $C$ and $\alpha$ are constants. This gives for the probability that at a distance $s$ from the surface a (primary) ion is still in the ionized state:

$$
P_{\mathbf{I}}(s)=\exp \left(-C \int_{-\infty}^{s} \exp (-\alpha s) \frac{\mathrm{d} t}{\mathrm{~d} s} \mathrm{~d} s\right)
$$

(see ref. 16), or

$$
\frac{\mathrm{d} \ln P_{\mathrm{I}}(s)}{\mathrm{d} t}=-C \exp (-\alpha s) \text {. }
$$

This model is based on the assumption of a structureless, smooth surface. However, the characteristic collision processes find their origin in the atomic structure of the surface; therefore it is reasonable to introduce this structure in the neutralization processes. In our model eq. (2) is replaced by

$$
f(s)=C \sum_{n} \exp \left(-\alpha r_{n}\right)
$$

where $r_{n}$ is the distance to the atom in the surface. Fig. 9 shows calculated values of $P_{\mathrm{I}}(\infty)$ as a function of the scattering angle for different values of $\alpha$ and fixed value of $C$. In the calculations Abrahamson's potential parameters were used to solve the equation of motion in the computer model $\left(\phi=30^{\circ}, 1 \mathrm{keV} \mathrm{Ne}^{+} \rightarrow \mathrm{Cu}\right)$. The upper part of each curve corresponds to reflection of the ion mainly on one atom, while the lower part corresponds to reflection mainly on two atoms. Multiplication of such an ion probability distribution with the density distribution of fig. 7 , gives the ion density distribution as a function of scattering angle (fig. 8 , curves $d$ and e). This distribution should coincide with the corrected experimental curve, which was obtained by subtracting the normalized tail of the distribution at $\phi=10^{\circ}$ from the distribution at $\phi=30^{\circ}$. The maximum of the corrected experimental peak intensity distribution (fig. 8, curve b) is best approximated for $\alpha=1.5$ and $2.0 \AA^{-1}$. For the value of $C$ used in fig. 9 , the remaining part of the experimental distribution is badly approximated. The slope of the calculated distributions is too small for small and large scattering angles. However, from the relation

$$
\ln P_{\mathrm{I}}(s)=-C \int_{-\infty}^{s} \sum_{n} \exp \left(-\alpha s_{n}\right) \frac{\mathrm{d} t}{\mathrm{~d} s} \mathrm{~d} s
$$

it is apparent that a change of the value of $C$ influences the slope of the calculated $P_{\mathrm{I}}(\infty)$ distribution for mainly single collisions.

With a suitable value of $C$ a good approximation may be found for the experimental peak intensity distribution of the single collision. However, for the value of $C$ adjusted to the experimental distribution of the single collisions (at $\alpha=1.5$ and $2 \AA^{-1}$ ), the cal- 


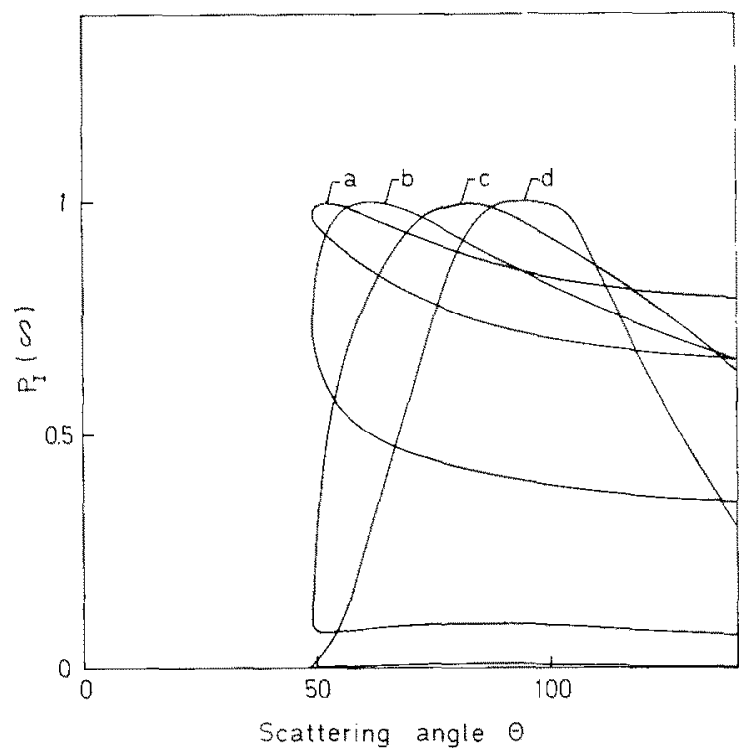

Fig. 9. The probability that $\mathrm{Ne}^{+}$persists as an ion after scattering on $\mathrm{Cu}(110)$ versus scattering angle $\left(\phi=30^{\circ}\right)$, calculated for different values of $\alpha$ and fixed value of $C\left(3.4 \times 10^{15} \mathrm{sec}^{-1}\right)$.

\begin{tabular}{lll} 
& $\alpha\left(\AA^{-1}\right)$ & $P_{1 \max }$ \\
\hline (a) & 3.0 & 0.5939 \\
(b) & 2.0 & 0.1674 \\
(c) & 1.5 & 0.0158 \\
(d) & 1.0 & $0.134 \times 10^{-4}$
\end{tabular}

All curves are normalized with respect to $P_{1} \max$.

culated distribution of the multiple scattering peak appears to be lower than the corresponding experimental distribution. For the most favourable values of $C$ the calculated distributions are plotted in fig. 8 (curves $d$ and e). The agreement with the experimental distribution (curve b) is still not complete. The best agreement between our experimental and calculated results will be found for $\alpha$ between 1.5 and $2.0 \AA^{-1}$ and $C$ between $3.4 \times 10^{15}$ and $9.4 \times 10^{15} \mathrm{sec}^{-1}$.

In table 1 these values are compared with literature data. In comparison with Hagstrum's results narrower limits for the values of the parameter $\alpha$ and $C$ are obtained. 
Table 1

Neutralization parameters

\begin{tabular}{|c|c|c|c|c|c|}
\hline \multirow[t]{2}{*}{$\alpha\left(\AA^{-1}\right)$} & \multicolumn{2}{|c|}{ Hagstrum [16] } & \multicolumn{2}{|c|}{ Van der Weg $[8,9]$} & \multirow{2}{*}{$\frac{\text { This work }}{2}$} \\
\hline & 2 & 5 & 2 & 1.5 & \\
\hline $\begin{array}{l}\mathrm{Ne}, C\left(\mathrm{~s}^{-1}\right) \times 10^{-15} \\
\mathrm{Ar}, C\left(\mathrm{~s}^{-1}\right) \times 10^{-15}\end{array}$ & $\begin{array}{r}48 \\
110\end{array}$ & $\begin{array}{l}1.6 \times 10^{5} \\
2.3 \times 10^{6}\end{array}$ & 0.24 & 3.4 & 9.4 \\
\hline
\end{tabular}

\section{Discussion and conclusion}

The parameters of an interatomic potential may be deterınined from the peak intensity distribution as a function of scattering angle (fig. 4) rather than from the scattering loops. This determination is improved by using the peak intensity distribution as a function of angle of incidence (fig. 6). For $1 \mathrm{keV} \mathrm{Ne}^{+} \rightarrow \mathrm{Cu}(110)$ the best agreement between experiment and model was found for $b=3.7 \AA^{-1}$ and $A>1.05 \times 10^{-15} \mathrm{~J}$ as parameters for the interatomic potential. In contrast with the results of Heiland et al. [5] for $\mathrm{Ar}^{+} \rightarrow \mathrm{Ni}$ the absolute values of the energy of the peaks are in good agreement.

It should be noted that in our model thermal vibrations are not considered. Consequently $\theta_{\min }$ and $\theta_{\max }$ as calculated from our model are too large and too small respectively when compared to experimental results. Probably $b$ is still too large and $A$ too small. A better approximation of the parameters may be expected by inclusion of thermal vibrations in the model.

From the peak intensity distributions it should be possible to determine the upper and lower values of the parameters in eq. (5) for the neutralization probability. However, for a more accurate determination of these parameters more information is needed about (i) the number of surface defects as a function of angle of incidence and (ii) the influence of thermal vibrations. Our experimental results at $\phi=30^{\circ}$ (figs. 4 and 8 ) were corrected for surface defects by subtracting the normalized tail of the distribution at $\phi=10^{\circ}$. Whether this is permitted will depend on the number of defects and the neutralization probability at these defects as a function of the angle of incidence. The peak intensity distributions should be measured at low energy and low primary ion currents to keep the influence of defects as small as possible. The influence of thermal vibrations on peak intensity distributions has been investigated by others [11]. Our experimental set-up does not allow this effect to be investigated. 


\section{Acknowledgement}

This work is part of the research program of the Stichting voor Fundamenteel Onderzoek der Materie (FOM) and was made possible by the financial support from the Nederlandse Organisatie voor Zuiver Wetenschappelijk Onderzoek (Netherland Organization for the Advancement of Pure Research).

\section{References}

[1] E.P.Th. Suurmeyer and A.L. Boers, Surface Sci. 43 (1974) 309.

[2] V.E. Yurasova, V.I. Shulga and D.S. Karpuzov, Can. J. Phys. 46 (1968) 759.

[3] V.M. Kivilis, E.S. Parilis and N. Yu. Turaev, Soviet Phys.--Dokl. 12 (1967) 328.

[4] E.S. Maskova and V.A. Molchanov, Radiation Effects 13 (1972) 183;

I.N. Evdokina, E.S. Maskova and V.A. Molchanov, Soviet Phys.-Dokl. 14 (1969) 467.

[5] W. Heiland, H.G. Schäffler and E. Taglauer, Surface Sci. 35 (1973) 381;

E. Taglauer and W. Heiland, Surface Sci. 33 (1972) 27.

[6] E.S. Parilis, in: Proc. 7th Intern. Conf. Phenomena in Ionized Gases, Belgrado, 1965, p. 129.

[7] H.D. Hagstrum, Phys. Rev. 123 (1961) 758.

[8] W.F. van der Weg, D.J. Bierman and D. Onderdelinden, Physica 44 (1969) 161.

[9] W.F. van der Weg and D.J. Bierman, Physica 44 (1969) 177.

[10] V.M. Kivilis, E.S. Parilis and N.Yu. Turaev, Soviet Phys. -Dokl. 15 (1970) 587.

[11] L.K. Verhey and A.L. Boers, in: Proc. 5 th Intern. Conf. on Atomic Collisions in Solids, Gatlinburg, U.S.A., 1973.

[12] S.H.A. Begemann and A.L. Boers, Surface Sci. 30 (1972) 134.

[13] S.H.A. Begemann, L.K. Verhey and A.L. Boers, Surface Sci. 35 (1973) 380.

[14] A. van Veen and J. Haak, Phys. Letters 40 A (1972) 398.

[15] A.A. Abrahamson, Phys. Rev. 178 (1969) 76.

[16] H.D. Hagstrum, Phys. Rev. 96 (1954) 336.

[17] W. Heiland, H.G. Schäffler and E. Taglauer, paper presented at the 5th Intern. Conf. on Atomic Collisions in Solids, Gatlinburg, U.S.A., 1973.

[18] W. Heiland and E. Taglauer, Radiation Effects 19 (1973) 1. 